\title{
Incidental identification of balanced translocation carrier patients through comprehensive chromosome screening of IVF-derived blastocysts
}

\author{
Nathan R. Treff • Eric J. Forman • Mandy G. Katz-Jaffe • \\ William B. Schoolcraft • Brynn Levy • Richard T. Scott Jr
}

Received: 18 March 2013 / Accepted: 2 May 2013 / Published online: 31 May 2013

(C) The Author(s) 2013. This article is published with open access at Springerlink.com

\begin{abstract}
Purpose To demonstrate that translocation carrier patients can be identified by analysis of chromosomes in preimplantation human embryos.

Methods A report of 3 cases in which multiple embryos were found to possess consistent segmental imbalances by CCS. The parents then had a conventional karyotype performed.
\end{abstract}

Capsule Carriers of balanced translocations are at increased risk of infertility due to the production of unbalanced gametes. While carriers are most often identified through family history, a history of recurrent pregnancy loss or genetic analysis of an unbalanced fetus, this study describes 3 cases where incidental identification resulted from having performed comprehensive chromosome screening on IVF-derived embryos.

N. R. Treff $(\triangle) \cdot$ E. J. Forman $\cdot$ R. T. Scott Jr

Reproductive Medicine Associates of New Jersey,

111 Madison Ave,

Morristown, NJ 07960, USA

e-mail: ntreff@rmanj.com

N. R. Treff • E. J. Forman • R. T. Scott Jr

UMDNJ-Robert Wood Johnson Medical School,

125 Paterson Street,

New Brunswick, NJ 08901, USA

N. R. Treff

Rutgers-The State University of New Jersey, 145 Bevier Road, Piscataway, NJ 08854, USA

M. G. Katz-Jaffe • W. B. Schoolcraft

Colorado Center for Reproductive Medicine, 10290 Ridge Gate, Circle Lone Tree, CO 80124, USA

B. Levy

Department of Pathology \& Cell Biology, Columbia University Medical Center \& the New York Presbyterian Hospital, New York, NY 10032, USA
Results In each case, parental karyotyping revealed the presence of an otherwise unknown balanced translocation. Original blastocyst CCS results were then reinterpreted to consider the presence of unbalanced derivative chromosomes and to modify the diagnosis of embryos eligible for transfer.

Conclusions It is possible to identify patients that are carriers of balanced translocations through the analysis of chromosomes in their IVF-derived embryos. Given that translocation carrier screening is not routinely performed, the growing use of CCS may facilitate discovery and provide both an etiology of reproductive failure and an improved more focused treatment strategy going forward. Future work will involve a large retrospective study to define the sensitivity and frequency of detection using this methodology.

Keywords Balanced translocation · SNP microarray · Quantitative real-time PCR · Aneuploidy · Comprehensive chromosome screening

\section{Introduction}

Carriers of balanced translocations are at an increased risk for miscarriage and producing offspring with congenital abnormalities and mental retardation, as a result of unbalanced segregation during gametogenesis [1]. Although individuals with infertility may have a higher risk of carrying a balanced translocation relative to the general population [2], carrier screening is not routinely performed without a more definitive indication such as recurrent pregnancy loss [3, 4], where approximately $0.5-5 \%$ of couples carry a balanced rearrangement [5]. Most often, patients carrying a translocation are identified after a miscarriage when genetic analysis of 
the products of conception reveals segmental chromosome imbalances. In those situations, parental karyotyping is ordered. Other individuals may have a family history leading to their referral for karyotyping prior to attempting conception. Once identified, in vitro fertilization (IVF) with preimplantation genetic diagnosis is a highly effective option for carriers of balanced translocations [6, 7]. However, to our knowledge, the identification of segmental imbalances in preimplantation embryos has not been reported as a source of information leading to the identification of individuals carrying a balanced translocation. This report describes 3 such cases where incidental findings following comprehensive chromosome screening (CCS) in IVF-derived embryos led to the identification of patients carrying balanced translocations.

\section{Materials and methods}

\section{CCS}

Patients indicated for CCS had IVF with blastocyst biopsy and either fresh embryo transfer or vitrifcation as previously described [8]. Trophectoderm biopsies were evaluated using a previously validated method of either SNP microarray-based [9] or quantitative realtime (q)PCR-based [10] CCS. Cases in which at least 2 embryos where observed to potentially possess segmental aneuploidies within the same genomic regions were identified as indicated for parental karyotyping.

\section{Conventional karyotyping}

G-banding was performed on each couple at one of 3 commercial reference laboratories; LabCorp Molecular Biology and Pathology (North Carolina), Columbia University Medical Center Campus of the New York Presbyterian Hospital (New York), or Integrated Genetics-Esoterix Genetic Laboratories, LLC (New Mexico). In each case, once a parental karyotype confirmed a balanced translocation carrier status, embryo CCS results were reinterpreted accordingly.

\section{Results}

In 3 cases, a balanced translocation carrier status was confirmed by conventional karyotyping after IVF-derived embryo based CCS indicated the possibility (Fig. 1). In each case, the original CCS results were reinterpreted to assign additional predictions of the inheritance of translocated chromosomes for each embryo (Table 1).

Case 1 was a 37 year old gravida 1 para 1 who presented with longstanding secondary infertility with a new partner. The male partner was a 48 year old with prior paternity in a previous relationship who was found to have severe oligospermia on repeat semen analyses. The couple's existing child was phenotypically normal. The patient underwent an IVF stimulation with final oocyte maturation achieved with leuprolide acetate due to risk of ovarian hyperstimulation (OHSS). A total of 58 oocytes were retrieved, of which 38 underwent ICSI and 33 fertilized normally. Due to the OHSS risk, nine blastocysts underwent trophectoderm biopsy and vitrification. After SNP microarray analysis, 3 embryos were predicted to be aneuploid. Four of the embryos had consistent segmental imbalances on chromosome 3. A conventional karyotype of the parents was ordered and confirmed a maternal balanced translocation, 46,XX,t(3;16)(p25.3; q24.3). When viewed in isolation, these segmental imbalances would not have altered the overall copy number call for chromosome 3. However, once a balanced translocation was documented on conventional karyotype, the CCS results were reinterpreted using a previously established analysis protocol for translocation carriers [6]. After re-analysis, there were 4 embryos predicted to be balanced or normal, one of which was warmed for frozen embryo transfer and she currently has an ongoing singleton pregnancy.

Case 2 was a 39 year old nulligravida who presented with longstanding unexplained infertility. She underwent 2 cycles of controlled ovarian hyperstimulation and timed intrauterine insemination without resulting in a pregnancy. She then underwent an IVF stimulation with planned CCS using a GnRH agonist downregulation protocol. She had 32 oocytes retrieved, of which 25 underwent ICSI and 21 normally fertilized. Four blastocysts were biopsied and vitrified. On subsequent qPCR analysis, 2 were predicted to be aneuploid $(47, \mathrm{XX},+22 ; 46, \mathrm{XY},-10,+13)$. One embryo was predicted to be euploid and the data on the forth embryo was determined to be non-concurrent based on previously established thresholds. She underwent another cycle in an attempt to accrue additional euploid blastocysts for future frozen transfer. In the next cycle, with a similar protocol, she produced 8 blastocysts that underwent trophectoderm biopsy. On analysis of the embryos, there were consistent segmental imbalances involving the distal regions of chromosomes 10 and 13. These observations prompted a recommendation for parental chromosome analysis which revealed a maternal balanced translocation, 46,XX,t(10;13)(q24.3;q12.1). In light of this finding, none of the embryos were suitable for transfer as 2 were aneuploid and 6 showed evidence of carrying unbalanced translocation derivative chromosomes. Furthermore, re-analysis of the qPCR data from the first IVF cycle revealed that the previously predicted normal and nonconcurrent embryos both carried the unbalanced translocation. The patient underwent a final IVF cycle, this time at age 40, with additional Taqman PCR primers for the translocation so that at least 4 assays on each side of the breakpoints for each of the two chromosomes involved in 


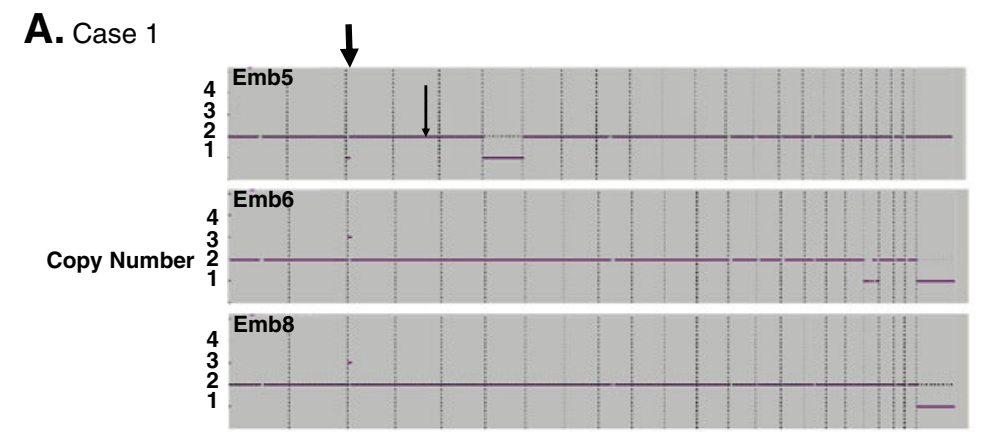

\section{Case 1 46,XX,t(3;16)(p25.3;q24.3)}
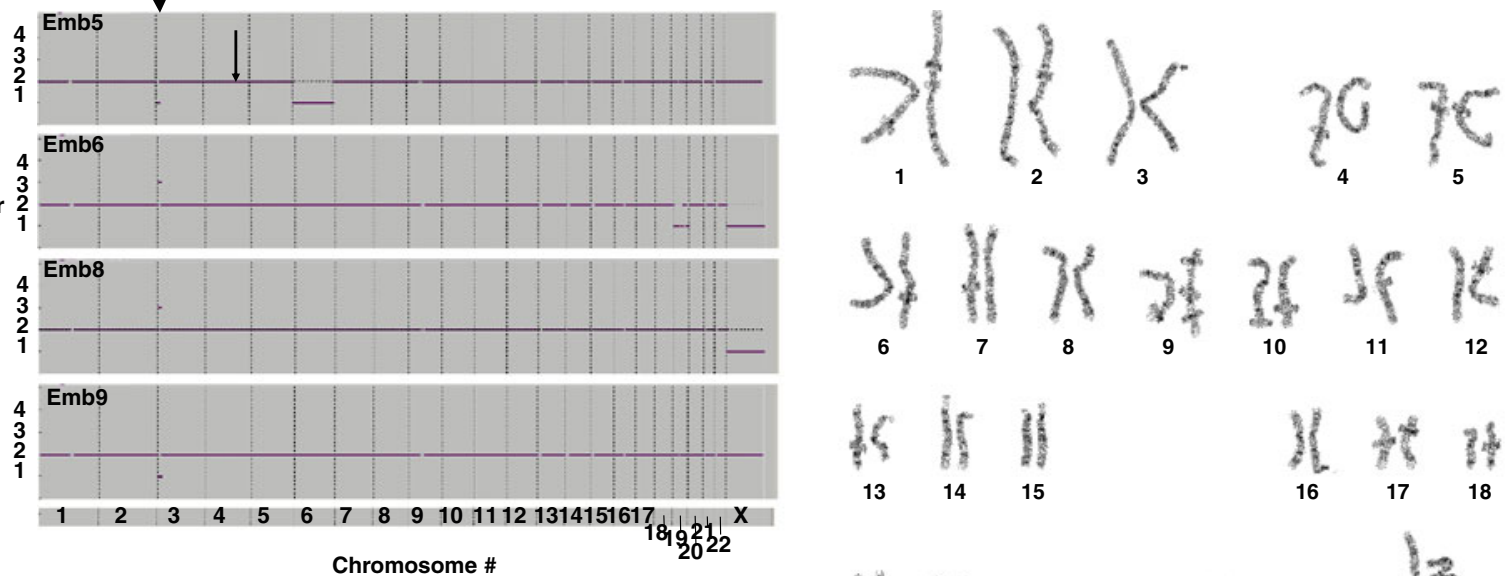

\section{B. Case 2}

\section{Emb2}

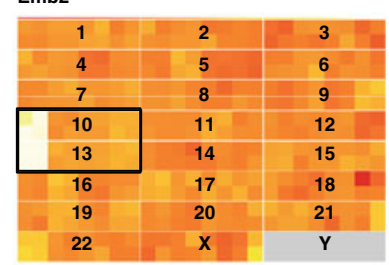

Emb5

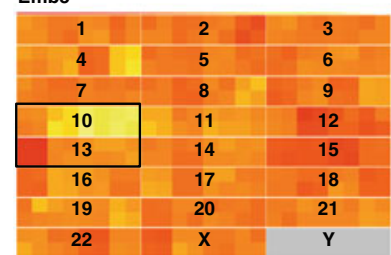

C. Case 3

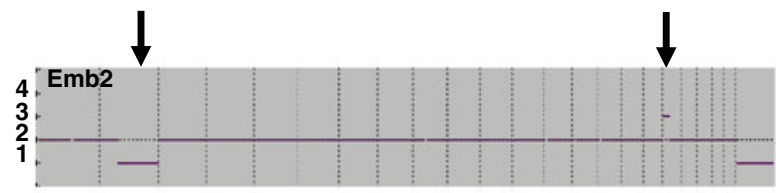

Copy Number
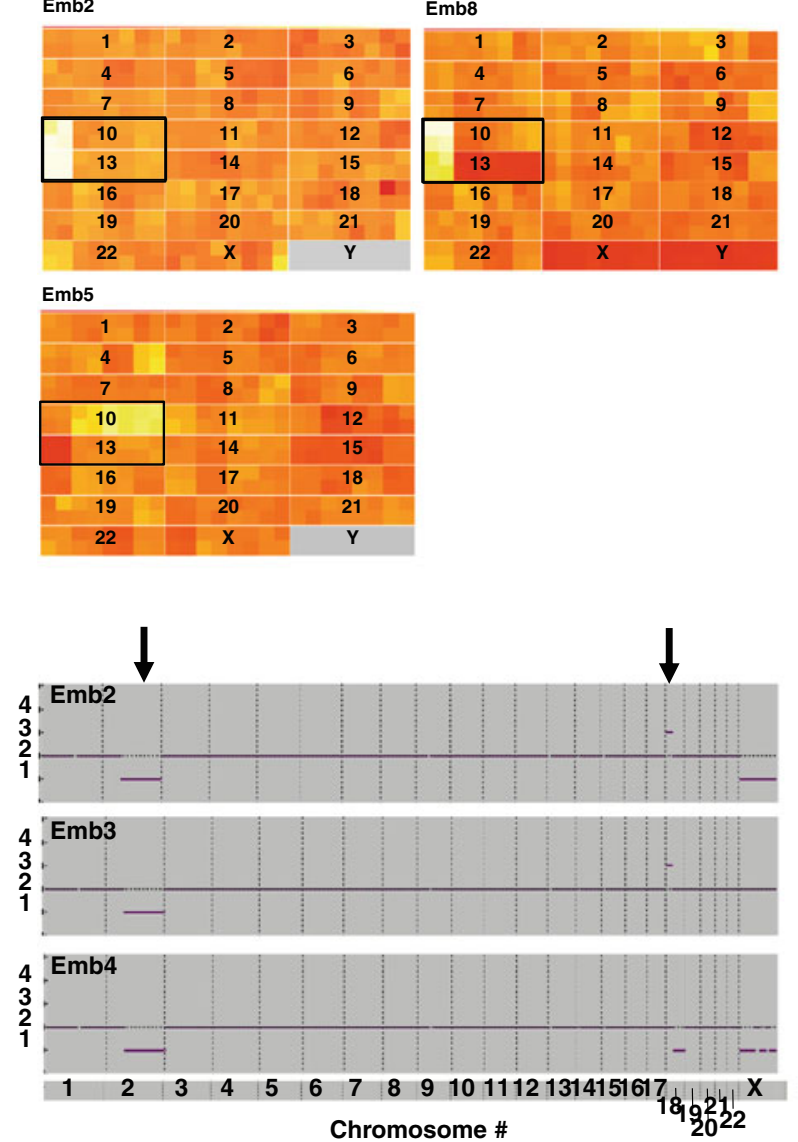

$\frac{1}{4}$ है
1920

E.

Case 2 46,XX,t(10;13)(q24.3;q12.1)

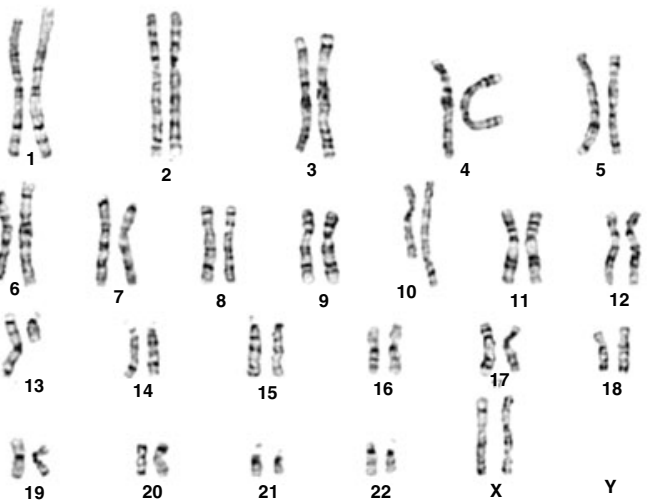

F.Case 3 46,XY,t(2;18)(p13.1; 12.2$)$
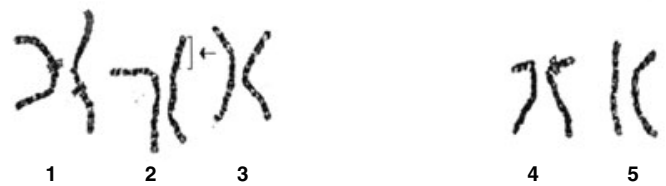

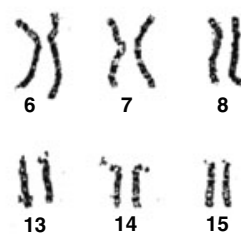

$\begin{array}{ll}18 & \$ 1 \\ 19 & 20\end{array}$

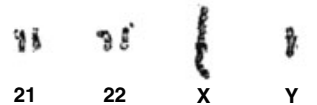

Fig. 1 Results of embryo CCS (a-c) and parental karyotyping (d-f) for 3 cases reported in this study. Black arrows indicate regions of imbalance in SNP array based CCS plots (a and $\mathbf{c})$, while black boxes highlight chromosomes with segmental imbalance in qPCR based CCS heatmaps (b). In the qPCR heatmaps, the lowest copy number (zero) is indicated by the most intense red color while the highest copy number (4.6) is indicated by a white color. Copy number changes are indicated by changes in color intensity from red-orange-yellow-white with an orange color indicating a copy number of 2 , red-like color indicating reduced copy number relative to 2 , and yellow-like color indicating increased copy number relative to 2. Grey indicates no amplification. The heatmaps are organized (from left to right and top to bottom) from chromosome 1 on the top left to chromosome $\mathrm{Y}$ on the bottom right, with each chromosome having 4 loci evaluated and 4 replicate reactions per loci (16 total data points per chromosome and 384 data points per embryo) 
Table 1 Embryo CCS results of 3 cases leading to an indication and identification of a parent with a balanced translocation

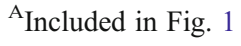

${ }^{\mathrm{B}}$ Diagnosis impacted

\begin{tabular}{|c|c|c|c|}
\hline Case No. & Embryo No. & Original CCS predicted karyotype & Revised CCS predicted karyotype \\
\hline \multirow[t]{9}{*}{1} & 1 & $46, X X$ & $46, \mathrm{XX}$ or $46, \mathrm{XX}, \mathrm{t}(3 ; 16)(\mathrm{p} 25.3 ; \mathrm{q} 24.3)$ \\
\hline & 2 & $46, X X$ & $46, \mathrm{XX}$ or $46, \mathrm{XX}, \mathrm{t}(3 ; 16)(\mathrm{p} 25.3 ; \mathrm{q} 24.3)$ \\
\hline & 3 & $46, X Y$ & $46, X Y$ or $46, X Y, t(3 ; 16)(p 25.3 ; q 24.3)$ \\
\hline & 4 & $47, X X,+16$ & $47, \mathrm{XX},+16$ \\
\hline & $5^{\mathrm{A}}$ & $45, X X,-6$ & $45, \mathrm{XX}, \operatorname{der}[3] \mathrm{t}(3 ; 16)(\mathrm{p} 25.3 ; \mathrm{q} 24.3),-6$ \\
\hline & $6^{\mathrm{A}}$ & $45, X Y,-19$ & $45, \mathrm{XY}, \operatorname{der}(16) \mathrm{t}(3 ; 16)(\mathrm{p} 25.3 ; \mathrm{q} 24.3),-19$ \\
\hline & 7 & $46, \mathrm{XX}$ & $46, \mathrm{XX}$ or $46, \mathrm{XX}, \mathrm{t}(3 ; 16)(\mathrm{p} 25.3 ; \mathrm{q} 24.3)$ \\
\hline & $8^{\mathrm{A}}$ & $46, X Y$ & $46, X Y, \operatorname{der}(16) \mathrm{t}(3 ; 16)(\mathrm{p} 25.3 ; \mathrm{q} 24.3)^{\text {В }}$ \\
\hline & $9^{\mathrm{A}}$ & $46, X X$ & $46, \mathrm{XX}, \operatorname{der}[3] \mathrm{t}(3 ; 16)(\mathrm{p} 25.3 ; \mathrm{q} 24.3)^{\mathrm{B}}$ \\
\hline \multirow[t]{12}{*}{2} & 1 & $47, X X,+22$ & $47, \mathrm{XX},+22$ \\
\hline & $2^{\mathrm{A}}$ & $46, \mathrm{XX}$ & $47, \mathrm{XX},+\operatorname{der}(13) \mathrm{t}(10 ; 13)(\mathrm{q} 24.3 ; \mathrm{q} 12.1)^{\mathrm{B}}$ \\
\hline & 3 & nonconcurrent & $46, X Y, \operatorname{der}(13) \mathrm{t}(10 ; 13)(\mathrm{q} 24.3 ; \mathrm{q} 12.1)^{\mathrm{B}}$ \\
\hline & 4 & $46, X X,-10,+13$ & $46, X X,-10,+13$ \\
\hline & $5^{\mathrm{A}}$ & $47, \mathrm{XX},+10$ & $46, \mathrm{XX},+\operatorname{der}[10] \mathrm{t}(10 ; 13)(\mathrm{q} 24.3 ; \mathrm{q} 12.1),-13$ \\
\hline & 6 & $47, X Y,+13$ & $47, X Y,+13$ \\
\hline & 7 & $46, X X,+10,-13$ & $46, X X, t(10 ; 13)(q 24.3 ; q 12.1)$ \\
\hline & $8^{\mathrm{A}}$ & $45, \mathrm{XY},-13$ & $46, X Y, t(10 ; 13)(\mathrm{q} 24.3 ; \mathrm{q} 12.1)$ \\
\hline & 9 & $44, X Y,-14,-19$ & $44, X Y,-14,-19$ \\
\hline & 10 & $47, X Y,+10$ & $47, \mathrm{XY},+10$ \\
\hline & 11 & $45, X X,-16$ & $45, X X,-16$ \\
\hline & 12 & $47, \mathrm{XX},+10$ & $46, \mathrm{XX},+\operatorname{der}[10] \mathrm{t}(10 ; 13)(\mathrm{q} 24.3 ; \mathrm{q} 12.1),-13$ \\
\hline \multirow[t]{4}{*}{3} & 1 & $46, X X$ & $46, \mathrm{XX}$ or $46, \mathrm{XX}, \mathrm{t}(2 ; 18)(\mathrm{p} 13.1 ; \mathrm{q} 12.2)$ \\
\hline & $2^{\mathrm{A}}$ & $45, X Y,-2$ & $46, \mathrm{XY},-2,+\operatorname{der}(18) \mathrm{t}(2 ; 18)(\mathrm{p} 13.1 ; \mathrm{q} 12.2)$ \\
\hline & $3^{\mathrm{A}}$ & $45, \mathrm{XX},-2$ & $46, \mathrm{XX},-2,+\operatorname{der}(18) \mathrm{t}(2 ; 18)(\mathrm{p} 13.1 ; \mathrm{q} 12.2)$ \\
\hline & $4^{\mathrm{A}}$ & $44, X Y,-2,-18$ & $45, \mathrm{XY},-2, \operatorname{der}(18) \mathrm{t}(2 ; 18)(\mathrm{p} 13.1 ; \mathrm{q} 12.2)$ \\
\hline
\end{tabular}

the translocation were included. Ten blastocysts underwent biopsy and qPCR, however all were unbalanced. The patient finally delivered after proceeding with oocyte donation.

The third patient presented as a 38 year old with primary infertility. Her partner was 41 years old and found to have teratozoospermia on semen analysis. She underwent an IVF cycle with a GnRH antagonist protocol and 12 mature oocytes were retrieved, of which 9 fertilized normally with ICSI. She produced 4 blastocysts that had trophectoderm biopsy performed. SNP microarray analysis revealed 1 euploid embryo and 3 embryos with a pattern of segmental imbalance on the distal regions of chromosomes 2 and 18 that was consistent with an unbalanced translocation. Parental chromosome analysis was ordered and revealed a paternal balanced translocation, 46,XY,t $(2 ; 18)(\mathrm{p} 13.1 ; \mathrm{q} 12.2)$. The single predicted euploid or balanced embryo was transferred and led to the delivery of a healthy female.

\section{Discussion}

This study has demonstrated that it is possible to use IVFderived embryo CCS analysis to identify certain patients at risk of carrying a balanced translocation. In each case, the discovery provided both an etiology for reproductive failure and a more focused treatment strategy. Furthermore, in the 3 cases reported here, 3 embryos originally found to be suitable for transfer based on criteria for assessing only whole chromosome aneuploidy were subsequently predicted to possess an unbalanced karyotype given the newly obtained translocation carrier status of the parents. The refinement of the original predicted karyotype in these cases may have averted a potential miscarriage or possibly the delivery of an affected child.

With the growing use of CCS to improve the success of IVF, it may be useful to establish criteria for recommending parental karyotyping based on observations made in embryos as demonstrated in this case report series. To this end, future work will involve a retrospective study of a large number of CCS cases in order to develop such criteria and determine sensitivity to and frequency of detecting cases indicated for translocation carrier screening. Results will be influenced by multiple factors including the CCS platform utilized (q-PCR or microarray) and its concomitant diagnostic resolution (i.e. limits of detection of small segmental aneuploidies associated with unbalanced translocations), as well as having a sufficient number of embryos 
evaluated within each case in order to observe imbalances within the same segment in more than one embryo. Given the demonstrated ability of CCS to improve single embryo transfer outcomes [11], the indications for CCS may be expanding. Patients without a prior indication for conventional karyotyping are increasingly using this technology. Nonetheless, this strategy is not necessarily meant to replace conventional karyotyping of indicated patients since CCS may not be indicated for all patients undergoing IVF. Instead, once a couple has already undergone an IVF cycle using CCS, it may be possible to evaluate whether parental translocation screening is indicated based upon observations made in the embryos produced.

\section{Conflict of interest None.}

Open Access This article is distributed under the terms of the Creative Commons Attribution License which permits any use, distribution, and reproduction in any medium, provided the original author(s) and the source are credited.

\section{References}

1. Neri G, Serra A, Campana M, Tedeschi B. Reproductive risks for translocation carriers: cytogenetic study and analysis of pregnancy outcome in 58 families. Am J Med Genet. 1983;16:53561.
2. Clementini E et al. Prevalence of chromosomal abnormalities in 2078 infertile couples referred for assisted reproductive techniques. Hum Reprod. 2005;20:437-42.

3. Medicine, P.C.o.t.A.S.f.R. Evaluation and treatment of recurrent pregnancy loss: a committee opinion. Fertil Steril. 2012;98:1103-11.

4. Crosignani PG, Rubin BL. Optimal use of infertility diagnostic tests and treatments. The ESHRE Capri Workshop Group. Hum Reprod. 2000;15:723-32.

5. Gardner RJM, Sutherland GR, Shaffer LG. Chromosome abnormalities and genetic counseling. 4th ed. New York: Oxford University Press; 2012.

6. Treff NR et al. Single nucleotide polymorphism microarray-based concurrent screening of 24-chromosome aneuploidy and unbalanced translocations in preimplantation human embryos. Fertil Steril. 2011;95(1606-1612):e1601-2.

7. Treff NR et al. Use of single nucleotide polymorphism microarrays to distinguish between balanced and normal chromosomes in embryos from a translocation carrier. Fertil Steril. 2011;96(1):e58-65.

8. Forman EJ et al. Single embryo transfer with comprehensive chromosome screening results in improved ongoing pregnancy rates and decreased miscarriage rates. Human reproduction (Oxford, England). 2012;27:1217-22.

9. Treff NR, Su J, Tao X, Levy B, Scott Jr RT. Accurate single cell 24 chromosome aneuploidy screening using whole genome amplification and single nucleotide polymorphism microarrays. Fertil Steril. 2010;94:2017-21.

10. Treff NR et al. Development and validation of an accurate quantitative real-time polymerase chain reaction-based assay for human blastocyst comprehensive chromosomal aneuploidy screening. Fertil Steril. 2012;97:819-824.e812.

11. Forman EJ et al. In vitro fertilization with single euploid blastocyst transfer: a randomized controlled trial. Fertil Steril In Press 2013. 